\title{
How can we fine-tune nanoparticles to improve drug delivery?
}

\author{
“...nanoparticles have tunable surface chemistry and it is this quality \\ that allows for intriguing opportunities to overcome issues of drug \\ resistance in target cells..."
}

First draft submitted: 1 March 2017; Accepted for publication: 14 March 2017; Published online: 21 July 2017

Keywords: conjugation $\bullet$ drug delivery $\bullet$ nanoparticles $\bullet$ reliable linkers $\bullet$ stimuli-responsive

"Multiple attempts have been taken to finetune nanoparticles to improve its therapeutic effciency of drug deliveries, including utilizing conjugated targeting ligands, rational design of reliable linkers between drug and carriers, and integration of the nanoparticle and drugs into different stimulus-response systems."

Drug-delivery systems aim to administer pharmaceutical compounds at a set rate for a preordained length of time and to achieve a therapeutic effect in a continued manner. Major advances have been made in the area of drug delivery systems since the early 1960s, and they have advanced from macroscopic controlled drug delivery devices and implants, to the current era of nanotechnology we are experiencing today [1]. Nanotherapeutic delivery systems present themselves as one of the most promising of their kind. As a drug delivery concept in nanotechnology, it strives to develop nanoscale materials for selective infusion of therapeutic drugs or pharmaceutical compounds to specific cells. It consists of submicron-sized colloidal particles such as nanoparticles, micelles, liposomes and vesicles [2]. These sophisticated materials have shown compelling results in their ability to transport a diverse range of pharmaceutical compounds to various parts of the human system including cells, tissues and organs for sustained periods of time [3-5]. Many biological processes in our bodies rely heavily on subcellular units that exist in the nanoscale (i.e., virus, ribosomes and molecular motors). Therefore, nanoparticles in sufficiently small size could allow for direct contact with subcellular compartments which provide an opportunity to interfere with intracellular processes [6].

However, manufacturing suitable targeted nanoparticles in drug deliveries have its own sets of challenges. In the design of suitable nanoparticles that can effectively pass through different kinds of physiological barriers in order to target specific cells with sufficient drugs, factors such as physiological barriers need to be considered. These barriers impede the permeation or internalization of pharmaceutical compounds that are large in size or have unfavorable membrane properties. Therefore, multiple studies have investigated on ways to fine-tune nanoparticles to improve therapeutic efficiency of drug deliveries, including utilizing conjugated targeting ligands, rational design for linker cleavage between drug and carriers, and integration into stimulus-response systems.

\section{Approaches to enhanced therapeutic efficiency of nanoparticles \\ Conjugation of targeting ligands}

The specificity of drug-delivery efficacies in nanoparticles can be achieved by successfully conjugating cell-specific ligands onto the nanoparticle surface. For example, in oncology, nanoparticles can be designed to integrate targeting ligands which bind to receptors that are only expressed in tumorous cells and not healthy cells. Cancer-associated biomarker molecules include the following:

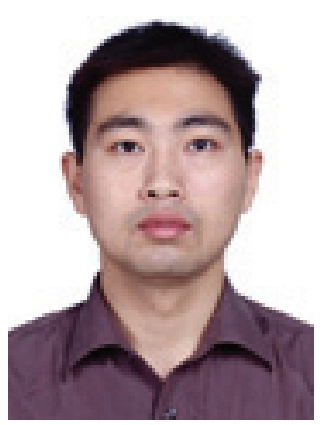

Zibiao Li

Institute of Materials Research \& Engineering, A*STAR (Agency for Science, Technology \& Research), 2 Fusionopolis Way, Innovis, \#08-03, 138634, Singapore lizb@imre.a-star.edu.sg 
families of vitamin receptors such as the FA vitamin B9 receptors (FAR- $\alpha$, FAR- $\beta$ ), riboflavin (vitamin B2) receptor and biotin receptor; the $\alpha v \beta 3$ integrin receptor; PSMA receptor; growth factor receptors like Her2, EGFR and FGFR; insulin and insulin-like receptors; a family of selectin protein molecules; and transferrin receptor. In the conjugation of the targeting ligands to nanoparticles, multiple ligand molecules will be attached to the nanoparticle surface [7]. This is because, in order for endocytic uptake of such targeted nanoparticles to occur, there needs to be multiple interactions existing concurrently between the interface of the cell surface receptor and targeted ligand pairs. This multivalent binding mechanism confers tight adhesion of the nanoparticle onto the targeted cell surface. This aspect is considered to be highly important during receptor-mediated uptake (endocytosis) of nanoparticles by the cell. Without it, the nanoparticles are bound weakly and have too short of a residence time to get taken up through the formation of coated pits. Therefore, by covalently conjugating many copies of a targeting ligand onto the nanoparticle surface, it maximizes these multivalent effects [8]. The challenge that we are facing is that the constitutive expression may still occur in healthy cells and therefore, tumorous and healthy cells may share the same ligand expression. A result of this is that, healthy cells can also absorb nanoparticles conjugated with targeted ligands when this happens, therapeutic efficiency of the administered drug is reduced. If the drug is toxic, cytotoxicity is another consequence.

\section{Prodrugs through linker cleavage}

Nanoparticles can also be attached to pharmaceutical compounds through covalent linkers for further enhancement in controlled drug release and therapeutic efficiency. Diseased cells have specific pathophysiological features and subcellular properties, and when certain conditions are in place, they trigger linker cleavage and cause the release of drugs. Linker type chemistries include ester, amide, disulfide, hydrazone, hypoxia-activated and self-immolative linkages [9].

Carboxylic-ester-based linker is formed between the nanoparticle and drug by having a pair of carboxylic acid or alcohol functional groups. Under physiological conditions, the ester bond is susceptible to hydrolysis process in vivo and this causes the drug to be released [10]. Amide linkers are formed with an amine or carboxylic acid group and are less susceptible to chemical hydrolysis compared with ester bond. Because of this stability, amide bonds normally do not hydrolysize under physiological conditions. Instead, a harsher environment like higher temperature or presence of strong acid or base cata- lyst is needed. Hydrazone-based linkers are terminated with acyl hydrazone, alkoxyl carbonyl hydrazone and benzenesulfonyl hydrazone. Many different classes of chemotherapeutic agents like DOX utilize hydrazone linkage for conjugation. Disulfide linkers undergo cleavage through disulfide exchange reaction or by obtaining thiol in an electrochemical reduction reaction. The chemical mechanism of cleaving is activated by endogenous thiol molecules. Because this cleavage mostly occurs in the cytoplasm after endocytosis, disulfide linkers are stable enough to navigate through extracellular environments to achieve targeted intracellular uptake. Hypoxia is the state where there is abnormally low oxygen supply in the cells and tissues. This method is a selective targeting of tumor cells by the use of prodrug and substrates that are activated only by oxidoreductase or enzymes that are active in hopoxic environment. This results in the release of drugs into tumorous cells. Self-immolation reaction is the sudden and rapid spontaneous intramolecular reaction that is activated by an applied external stimulus like enzymes, thiol-disulfide exchange, low $\mathrm{pH}$, bioreduction and light. Under these external stimuli, each of the triggering moiety is cleaved and this activates the free spacers and electronic cascade reaction or spontaneous cyclization, thereby releasing the drug.

\section{Stimulus-responsive nanocarriers}

To avoid the harmful effects of chemotherapeutic agents on healthy cells, these drugs must be delivered specifically to cancerous cells. This highly specific drug release mechanism can be controlled via stimulus responsive trigger on nanoparticles [11]. This allows the drug to target only specific cells and thus maintain a concentration above the therapeutic concentration for a particular length of time. The main and popular stimuli for remotely triggered systems are near infrared (NIR) light, ultrasound and alternating magnetic field (AMF) [12]. NIR light can be used to stimulate plasmonic metallic nanoparticles (i.e., gold nanoparticles [GNPs]). Surface plasmon resonance oscillations on GNPs results in strong absorption of NIR light. These nanoparticles then convert optical energy from NIR light into thermal energy, which is known as NIR-induced photothermia. This heat stress allows for tumor ablation and also temperature-dependent release of pharmaceutical drugs. Exposure of nanoparticles to ultrasound will lead to a temperature increase (ultrasound hyperthermia). Focused and directed ultrasound offers superb spatial and temporal precision as well as conjuring a physical force onto the target. These impacts could be used to trigger the release of drug molecules when GNPs are 
integrated with a thermoresponsive substances. AMF is able to produce local heating around magnetic nanoparticles, like iron oxide nanoparticles through hysteresis losses, known as magnetic hyperthermia. This heat stress can be remotely triggered on tumor cells, causing intracellular and extracellular degradation. Magnetic nanoparticles attached to drug molecules can also be integrated with thermosresponsive materials which can be induced to release its therapeutic contents upon exposure to AMF. In another aspect, in a diseased cell, the pathological microenvironment changes in $\mathrm{pH}$, concentration of glucose and reductive reagents, and the presence of specific enzyme [13,14]. These changes are manipulated in vivo to stimulate a trigger for drug release [15]. For example, $\mathrm{pH}$ in tumorous tissues are lower than health tissues [16,17]. The acidic environment in vivo can be exploited internally to stimulate a trigger release of a $\mathrm{pH}$-sensitive drug. The difference in the pathological microenvironments between healthy and tumor cells offers a way for tumor-targeted drug delivery [18].

\section{References}

1 Li Z, Loh XJ. Recent advances of using polyhydroxyalkanoate-based nanovehicles as therapeutic delivery carriers. Wiley Interdiscip. Rev. Nanom. Nanobiotechnol. doi:10.1002/wnan.1429 (2016) (Epub ahead of print).

2 Karim AA, Dou Q, Li Z et al. Emerging supramolecular therapeutic carriers based on host-guest interactions. Chem. Asian J. 11(9), 1300-1321 (2016).

3 Li Z, Tan BH, Lin T et al. Recent advances in stereocomplexation of enantiomeric PLA-based copolymers and applications. Prog. Polym. Sci. 62, 22-72 (2016).

$4 \quad$ Li Z, Swee LW. Functionalization of 2D transition metal dichalcogenides for biomedical applications. Mater. Sci. Eng. C Mater. Biol. Appl. 70, 1095-1106 (2017).

5 Li Z, Loh XJ. Water soluble polyhydroxyalkanoates: future materials for therapeutic applications. Chem. Soc. Rev. 44(10), 2865-2879 (2015).

6 Fan X, Jiang S, Li Z et al. Conjugation of poly(ethylene glycol) to poly(lactide)-based polyelectrolytes: an effective method to modulate cytotoxicity in gene delivery. Mater. Sci. Eng. C Mater. Biol. Appl. 73, 275-284 (2017).

7 Fan X, Zhang W, Hu Z et al. Facile synthesis of RGDconjugated unimolecular micelles based on a polyester dendrimer for targeting drug delivery. J. Mater. Chem. 5, 1062-1072 (2017).

8 Lu Y, Low PS. Folate-mediated delivery of macromolecular anticancer therapeutic agents. Adv. Drug Deliv. Rev. 64, 342-352 (2012).

\section{Conclusion}

Nanotherapeutic delivery strategies have great potential to overcome problems associated with normal drug delivery methods attributed to their improved pharmacokinetics and pharmacodynamics [19]. This is especially so in the area of anticancer therapeutics, where almost all chemotherapeutic agents suffer from one or more of such issues [20]. In general, nanoparticles have tunable surface chemistry and it is this quality that allows for intriguing opportunities to overcome issues of drug resistance in target cells and facilitate movement of drugs across barriers for a better therapeutic efficacy.

\section{Financial \& competing interests disclosure}

The author has no relevant affiliations or financial involvement with any organization or entity with a financial interest in or financial conflict with the subject matter or materials discussed in the manuscript. This includes employment, consultancies, honoraria, stock ownership or options, expert testimony, grants or patents received or pending, or royalties.

No writing assistance was utilized in the production of this manuscript. nanotherapeutic delivery systems. Chem. Rev. 115(9), 3388-3432 (2015).

10 Li Z, Yang J, Loh XJ. Polyhydroxyalkanoates: opening doors for a sustainable future. NPG Asia Mater. 8, e265 (2016).

11 Li Z, Yuan D, Jin G et al. Facile layer-by-layer self-assembly toward enantiomeric poly(lactide) stereocomplex coated magnetite nanocarrier for highly tunable drug deliveries. ACS Appl. Mater. Interfaces 8(3), 1842-1853 (2016).

12 Zhang P, Wang C, Zhao J et al. Near infrared-guided smart nanocarriers for microRNA-controlled release of doxorubicin/siRNA with intracellular ATP as fuel. ACS Nano 10(3), 3637-3647 (2016).

13 Li Z, Chee PL, Owh C et al. Safe and efficient membrane permeabilizing polymers based on PLLA for antibacterial applications. RSC Adv. 6, 28947-28955 (2016).

14 Li Z, Yuan D, Fan X et al. Poly(ethylene glycol) conjugated poly(lactide)-based polyelectrolytes: synthesis and formation of stable self-assemblies induced by stereocomplexation. Langmuir 31(8), 2321-2333 (2015).

15 Fan X, Chung JY, Lim YX et al. A review of adaptive programmable materials and their bio-applications. ACS Appl. Mater. Interfaces 8(49), 33351-33370 (2016).

16 Wu YL, Wang H, Qiu YK et al. PHB-based gels as delivery agents of chemotherapeutics for the effective shrinkage of tumors. Adv. Healthc. Mater. 5(20), 2679-2685 (2016).

17 Liow SS, Dou Q, Kai D et al. Long-term real-time in vivo drug release monitoring with AIE thermogelling polymer. Small doi:10.1002/smll.201603404 (2016) (Epub ahead of print). 
18 Fan X, Wang Z, Yuan D et al. Novel linear-dendriticlike amphiphilic copolymers: synthesis and self-assembly characteristics. Polym. Chem. 5, 4069-4075 (2014).

19 Fan X, Li Z, Loh XJ. Recent development of unimolecular micelles as functional materials and applications. Polym. Chem. 7, 5898-5919 (2016).
20 Hwang JY, Li Z, Loh XJ. Small molecule therapeutic-loaded liposomes as therapeutic carriers: from development to clinical applications. RSC Adv. 6, 70592-70615 (2016). 